\title{
Avaliação Fisioterapêutica da Força Muscular do Assoalho Pélvico na Mulher com Incontinência Urinária de Esforço após Cirurgia de Wertheim-Meigs: Revisão de Literatura
} Physiotherapeutic assessment of the Muscular Strength of the Pelvic Floor in Women with Urinary Incontinence after Wertheim-Meigs' Surgery: a Literature Review

Simone Mattos do Nascimento

\section{Resumo}

Introdução: as mulheres portadoras de câncer do colo do útero, nos estágios Ia, Ib e IIa, e adenocarcinoma cervical primário são submetidas à histerectomia radical de Wertheim-Meigs, que pode evoluir com alguns eventos adversos, entre eles a incontinência urinária de esforço (IUE), decorrente da fraqueza da musculatura do assoalho pélvico. A avaliação da força muscular do assoalho pélvico tem papel decisivo no tratamento fisioterápico da IUE. Objetivo: descrever os métodos fisioterapêuticos que podem ser utilizados para avaliar a força muscular do assoalho pélvico nas mulheres com IUE após cirurgia de Wertheim-Meigs. Método: o presente artigo consiste numa revisão bibliográfica, a qual pesquisou estudos publicados nas bases de dados Medline, Pubmed, Scielo, Lilacs e Bireme, no período de 1996 a 2007, em inglês e português. Conclusão: fundamentada nessa revisão, conclui-se que o método de avaliação funcional é um método eficaz e de fácil aplicabilidade, sendo por isso o mais utilizado nas avaliações fisioterapêuticas, na prática clínica.

Palavras-chave: Assoalho pélvico; Incontinência urinária de esforço; Histerectomia e Reabilitação

Pós-graduanda do Curso de Especialização em Gerontologia e Geriatria, Universidade Federal Fluminense (UFF); Pós-graduanda do Curso de Especialização em Fisioterapia em Oncologia, Instituto Nacional de Câncer (INCA)

Endereço para correspondência: Avenida Antônio Carlos, 8.300 - casa 5 - Pampulha - Belo Horizonte (MG), Brasil - CEP-31270-000. E-mail: simonemtn@uol.com.br 


\section{INTRODUÇÃO}

Atualmente, a histerectomia radical de WertheimMeigs é uma das abordagens terapêuticas recomendadas para o tratamento do câncer do colo do útero, segundo a International Federation of Gynecology and Obstetrics $(\text { FIGO })^{1}$. Diante da possibilidade do surgimento de incontinência urinária como efeito adverso a longo prazo, após sequela da histerectomia, faz-se necessário que as mulheres sejam alertadas sobre esse dano uroginecológico.

Define-se como incontinência urinaria de esforço (IUE) toda perda involuntária de urina, através de canal uretral íntegro, quando a pressão vesical excede a pressão uretral máxima, na ausência de atividade do músculo detrusor, sendo o tipo mais frequente de perda involuntária de urina na mulher, sincrônica ao esforço, espirro ou tosse ${ }^{2}$. O músculo detrusor é um músculo liso da parede da bexiga urinária que armazena certa quantidade de urina sem alterar a pressão (complacência vesical). Nesta fase, o músculo detrusor (bexiga), que está em repouso, produz, dentro da parede vesical, uma estimulação simpática dos receptores beta-adrenérgicos, causando o seu relaxamento durante a fase de enchimento. Em seguida, ocorre a estimulação simpática de receptores alfa-adrenérgicos no colo vesical e uretra, causando uma constrição, com consequente aumento da pressão. À medida que a bexiga continua se enchendo, os reflexos de micção tornam-se mais frequentes e causam contrações do músculo detrusor, a IUE ocorre na ausência de contração desse músculo da bexiga ${ }^{3}$.

A mulher portadora de IUE após histerectonia radical Wertheim-Meigs vivencia uma condição social constrangedora, ocasionando a exclusão social, e traz como consequência principal uma piora significativa na qualidade de vida. Essas mulheres, silenciosamente, têm redução da autoestima, tornando-se deprimidas, angustiadas e irritadas ${ }^{4}$.

A avaliação fisioterapêutica para a IUE deve incluir orientaçōes para a complementação diagnóstica que auxiliem na quantificação da perda urinária. Os métodos utilizados são o diário urinário, o teste de esforço, o teste do cotonete (Q-tip) e a prova do absorventes.

A avaliação da força muscular das fibras dos tipos I (30\% contração lenta) e II (70\% contração rápida) do assoalho pélvico lesionadas, após o procedimento cirúrgico citado, permite uma graduação inicial que determina um fisiodiagnóstico significativo, fundamentado por meio da utilização da avaliação funcional visual, do uso do perineômetro, dos cones vaginais, da eletroestimulação de superfície e do biofeedback. Dessa forma, o objetivo principal consistiu- se em descrever os métodos fisioterapêuticos que podem ser utilizados para avaliar a força muscular do assoalho pélvico nas mulheres com IUE após cirurgia de Wertheim-Meigs.

\section{METODOLOGIA}

O presente artigo consiste de uma revisão bibliográfica, tendo como proposta levantar os estudos que fizessem referência à avaliação da força muscular do assoalho pélvico, a partir de uma abordagem fisioterapêutica, publicados no período de 1994 a 2007, nos idiomas inglês e português, entre os quais estão livros e artigos de revistas disponíveis nas bases de dados nacionais e internacionais (Medline, Pubmed, Scielo, Lilacs e Bireme).

\section{MÉTODOS FISIOTERAPÊUTICOS PARA AVALIAÇãO DA FORÇA MUSCULAR DO ASSOALHO PÉLVICO}

\section{a) Avaliacão Visual}

A avaliação visual funcional mostra a visualização da genitália externa, verificando-se a presença ou ausência de contração voluntária visível da musculatura do assoalho pélvico após comando verbal, isto é, a observação do movimento do períneo para dentro durante a contração do músculo do assoalho pélvico $(\mathrm{MAP})^{6}$. Diante desse contexto, a visualização da contração da muscular do assoalho pélvico é baseada na graduação de 0 a 5 , a qual permite determinar a classificação funcional do MAP, conforme o quadro 1, no qual se referem os autores Bernardes et al. $(2000)^{5} \mathrm{e}$ Contreras Ortiz O, Coya Nuñez F, Ibañez G (1994) Baseados nesse procedimento, as pacientes foram avaliadas da seguinte maneira: Grau 0 - sem contração perineal visível, nem à palpação (ausência de contração); Grau 1 - sem contração perineal visível, contração reconhecível somente à palpação; Grau 2 - contração perineal fraca, contração fraca à palpação; Grau 3 contração perineal presente e resistência não opositora à palpação; Grau 4 - contração perineal presente e resistência opositora não mantida mais do que cinco segundos à palpação; e Grau 5 - contração perineal presente e resistência opositora mantida mais do que cinco segundos à palpação. O MAP com função deficiente ou inadequada é um fator etiológico relevante na ocorrência de IUE. Para fins de classificação, as portadoras dessa sintomatologia são aquelas que apresentam um grau de força de contração MAP igual a 1, 2, $3^{5}$.

O MAP é tradicionalmente avaliado por meio da palpação vaginal. A paciente permanece posicionada na postura ginecológica desnuda da cintura para baixo, seguida da percepção tatilpalpatória pelo teste digital, 
Quadro 1. Classificação funcional do assoalho pélvico de acordo com a contração voluntária

\begin{tabular}{|c|c|c|}
\hline Grau & $\begin{array}{c}\text { Função subjetiva } \\
\text { Visualização da contração } \\
\text { em posição ginecológica }\end{array}$ & À palpação \\
\hline 0 & Ausente & Ausente \\
\hline 1 & Ausente & Reconhecível \\
\hline 2 & Débil & Seconhecível \\
\hline 3 & Presente & Com resistência \\
\hline 4 & Presente & $\begin{array}{c}\text { Concia }<5 " \\
\text { (resistência não mantida) }\end{array}$ \\
\hline 5 & Presente & $\begin{array}{c}\text { Com resistência }>5 " \\
\text { (resistência mantida) }\end{array}$ \\
\hline
\end{tabular}

Fonte: Bernardes et al., 20005; Contreras Ortiz O, Coya Nuñez F, Ibañez G,1994 ${ }^{6}$

em que o examinador introduz o dedo indicador e o médio no introito vaginal. Hanh et al. (1996) ${ }^{7}$ deduziram que a palpação vaginal permitiu que as pacientes contraíssem a musculatura o mais fortemente possível, avaliando-se melhor as fibras do tipo II, de contração rápida, mais sensível à fadiga. Dessa forma, é possível averiguar a força muscular e classificar a paciente nas cinco categorias, conforme mostrado no Quadro 1.

A preferência pelo uso da classificação funcional do assoalho pélvico quando se solicita a contração voluntária se deu pela simplicidade e rapidez da técnica. A avaliação da contração voluntária é a chave para determinar a modalidade do tratamento, na escala 0 a 5 , mostrando a ausência e normalidade da força muscular, respectivamente. A mulher classificada com graus 0,1 , 2 não foi capaz de realizar a contração voluntária da musculatura do assoalho pélvico, apresentando nenhuma contração ou fraqueza muscular, quando, então, se recomenda como tratamento a eletroestimulação, biofeedback e/ou cones vaginais. Para as mulheres com graus $3,4,5$, indicam-se exercícios para a musculatura do assoalho pélvico ${ }^{8}$.

\section{b) Perineômetro}

Perineômetro é um manômetro da pressão que mede a habilidade do MAP para desenvolver o aperto vaginal ${ }^{9}$. Esse instrumento avalia o pico máximo de contração muscular do assoalho pélvico, visualizando no visor do aparelho em $\mathrm{cmH}_{2} \mathrm{O}$, que varia de 0 a $256 \mathrm{cmH}_{2} \mathrm{O}$; e com auxílio do cronômetro digital deve-se medir o tempo máximo de contração com esse método.

\section{c) Cones Vaginais}

A avaliação funcional do assoalho pélvico é realizada com cones vaginais, esses dispositivos são utilizados também no tratamento da $\mathrm{IUE}^{6}$. Quando introduzidos no canal vaginal, proporcionam um vigoroso biofeedback tátil e cinestésico, facilitando o assoalho pélvico a se contrair de forma reflexa na tentativa de retê- $-l^{10}{ }^{10}$. Os cones são dispositivos de tamanho igual e pesos diferentes que devem ser introduzidos e mantidos na vagina. De acordo com o peso que a paciente consegue manter o aparelho fixado na vagina, o peso é graduado em 0 (não mantém o peso de 25 gramas) até 5 (mantém o peso de 75 gramas); tais pesos são crescentes e numerados da seguinte maneira: $\mathrm{n}^{\circ} 1=20 \mathrm{~g} ; \mathrm{n}^{\circ} 2=32,5 \mathrm{~g} ; \mathrm{n}^{\circ} 3=45 \mathrm{~g}$; $\mathrm{n}^{\circ} 4=57,2 \mathrm{~g} ; \mathrm{n}^{\circ} 5=70 \mathrm{~g}^{6}$.

Para Belo et al. $(2005)^{11}$, a avaliação inicia-se com a introdução na vagina dos dispositivos mais leves, em decúbito dorsal com os quadris e joelhos fletidos, deixando-se o fio de náilon para fora para possibilitar a remoção do cone. Após o exame, a paciente é submetida a uma sequência crescente de esforços, que são padronizados de acordo com o Serviço de Fisioterapia (caminhar durante um minuto; subir/descer escadas durante 30 segundos; pular, agachar-levantar, tossir quatro vezes). Caso o dispositivo permaneça no canal vaginal, ele é, então, removido e insere-se o próximo de maior peso e assim sucessivamente, até que algum deles se exteriorize aos esforços. A classificação do grau de força do assoalho pélvico fez-se de acordo com o peso do cone sustentado no momento da avaliação da força muscular do assoalho pélvico das mulheres portadoras de IUE $^{10}$. Hanh et al. (1996) deduziram que as fibras do tipo I, responsáveis pela contração lenta, foram mais bem avaliadas pela utilização dos cones vaginais.

No Quadro 2, foi descrito a avaliação da musculatura do assoalho pélvico por meio dos cones vaginais.

\section{d) Eletromiografia de Superfície}

A eletroestimulação vaginal e retal é muito preconizada para a avaliação da força do músculo, principalmente quando o grau de força muscular for 
inferior a $3^{7}$. Para Nagib, et al. $(2005)^{12}$, a eletromiografia (EMG) do assoalho pélvico é uma avaliação que reproduz e pode predizer, de forma consistente, determinadas variáveis clínicas relacionadas (mobilidade da uretra, por exemplo) ao funcionamento dessa musculatura, por ser um método mais preciso para mensurar a integridade neuromuscular. Essa técnica utiliza um eletrodo que é capaz de medir a atividade voluntária das unidades motoras, em que eletrodos de superfície acoplados na região perineal são capazes de captar a atividade do MAP e da mucosa vaginal, além de fornecer informaçôes similares àquelas derivadas de eletrodos de agulha periuretral ${ }^{13}$. Na pré-avaliação de sete pacientes com IUE, verificou-se que $85 \%$ dos casos tinham força muscular igual a "0" e 14,3\% apresentaram força igual a "1"; após tratamento com EMG, as pacientes apresentaram, no máximo, uma força igual a $2^{6}$.

Quadro 2. Avaliação da musculatura do assoalho pélvico através dos cones vaginais

\begin{tabular}{|c|l|}
\hline Índice & \multicolumn{1}{|c|}{ Observação clínica } \\
\hline 0 & O peso número $1(25 \mathrm{~g})$ cai \\
\hline 1 & $\begin{array}{l}\text { O peso número } 1 \text { é mantido e o } \\
\text { número } 2(35 \mathrm{~g}) \text { cai }\end{array}$ \\
\hline 2 & $\begin{array}{l}\text { O peso número } 2 \text { é mantido e o } \\
\text { número } 3(45 \mathrm{~g}) \text { cai }\end{array}$ \\
\hline 3 & $\begin{array}{l}\text { O peso número } 3 \text { é mantido e o } \\
\text { número } 4(55 \mathrm{~g}) \text { cai }\end{array}$ \\
\hline 4 & $\begin{array}{l}\text { O peso número } 4 \text { é mantido e o } \\
\text { número } 5(65 \mathrm{~g}) \text { cai }\end{array}$ \\
\hline 5 & O peso número 5 é mantido \\
\hline
\end{tabular}

Fonte: Moreira, $2000^{10}$

\section{e) Biofeedback}

O biofeedback é bastante utilizado na reeducação do assoalho pélvico; é um instrumento que proporciona à paciente uma resposta, por meio de sinais luminosos, numéricos e sonoros, permitindo à paciente realizar a autoavaliação do MAP sobre o movimento que possa ser medido, ensinado e aprendido, para que ela tenha uma consciência maior da sua contração muscular, sendo um importante recurso a ser usado nos estágios iniciais da avaliação e do tratamento ${ }^{14}$.

O biofeedback de pressão utiliza-se de uma sonda inflável, colocada no interior da vagina, ou do ânus, se mulheres virgens. Quando é feita a contração, a variação de pressão dentro dessa sonda inflável é detectada pelo aparelho e mostrada à paciente. "É também útil para melhorar a visão sinestésica da região". O biofeedback de pressão é um recurso terapêutico dispendioso e, dependendo dos recursos oferecidos ou de sua origem, importado ou não, fica mais oneroso o equipamento ${ }^{15}$.

O biofeedback eletromiográfico, assim como o de pressão, tem um sensor que detecta a atividade elétrica que é liberada pelos músculos quando ocorre a contração. Quando a mulher faz a contração do MAP, esse sinal é detectado pelos sensores na derme, que o amplificam à resposta fisiológica e processam esse sinal de forma sonora ou visual para a paciente ${ }^{16}$. É muito difícil, para quem tem traumas na região ou uma atrofia muscular considerável, ter a percepção sinestésica desse movimento. O biofeedback fornece essa resposta, possibilitando uma avaliação direta pelo terapeuta e pela paciente ${ }^{17}$.

A avaliação do assoalho pélvico mostrou que a função muscular foi menos eficiente nas mulheres incontinentes 5 . O exame físico é primordial para classificar a disfunção muscular, de acordo com as escalas de força muscular e a aplicabilidade de um protocolo de tratamentos fisioterapêuticos, priorizando a normotonicidade vaginal.

\section{DISCUSSÃO E CONCLUSÃO}

Os estudos foram unânimes em relação à influência da avaliação da força muscular do assoalho pélvico antes e após o tratamento fisioterapêutico; nas mulheres com IUE, a graduação da força muscular do assoalho pélvico foi menos eficiente em comparação às continentes ${ }^{10}$.

No Quadro 3, foi elaborada uma comparação entre os métodos de avaliação da força muscular, enfatizando os pontos positivos e negativos.

O índice de confiança e a correlação das técnicas simples para medir a força do MAP incluíram a avaliação funcional visual, o perineômetro e os cones vaginais. $\mathrm{O}$ exame não mostrou qualquer variabilidade intrateste para palpação vaginal. $O$ perineômetro mostrou que a diferença absoluta entre a contração máxima força e intensa durante $5 \mathrm{~s}$ de contração foi inferior a $5,3 \mathrm{mmHg}$ e 4,5 $\mathrm{mmHg}$, respectivamente, com uma probabilidade de 0,95 . A contração máxima força e intensa à média de duração de 5 s contração foi bem correlacionada à palpação vaginal, mas apenas fracamente com os cones vaginais. A confiabilidade da medição força do MAP é mais elevada na palpação vaginal, seguida pelas mediçōes no perineômetro, e depois pelos cones vaginais ${ }^{22}$.

Os métodos de avaliação funcional visual e perimeômetro, para análise da reprodutibilidade do teste interavaliador, comparados com o sistema de classificação modificada Oxford de força muscular, apresentaram uma confiabilidade de 0,70 para avaliação 
Quadro 3. Comparação entre os métodos de avaliação da força muscular, enfatizando os pontos positivos e negativos

\begin{tabular}{|c|c|c|}
\hline Métodos & Pontos positivos & Pontos negativos \\
\hline $\begin{array}{l}\text { Avaliação } \\
\text { funcional visual }\end{array}$ & $\begin{array}{l}\text { - Utilizados para documentar } \\
\text { função do assoalho pélvico } \\
\text { Verifica-se a presença ou ausência } \\
\text { de contração voluntária visível do } \\
\text { MAP após comando verbal }\end{array}$ & $\begin{array}{l}\text { - A técnica varia de um fisioterapeuta para } \\
\text { outro, particularmente no que diz } \\
\text { respeito à resistência oposta e à duração } \\
\text { da contração }\end{array}$ \\
\hline Perineômetro & $\begin{array}{l}\text { - Permite a reprodutibilidade dos } \\
\text { resultados e objetividade do } \\
\text { exame }\end{array}$ & $\begin{array}{l}\text { - Requer certa experiência do profissional } \\
\text { para ser usado adequadamente }\end{array}$ \\
\hline Cones vaginais & $\begin{array}{l}\text { - } \quad \text { Atua como um feedback sensorial } \\
\text { - Método efetivo, de baixo custo e } \\
\text { pouco invasivo } \\
\text { - Permite graduar o grau força } \\
\text { muscular do MAP de acordo com } \\
\text { o peso do cone sustentado }\end{array}$ & $\begin{array}{l}\text { - As mulheres muito obesas com introito } \\
\text { pequeno, geralmente, têm dificuldade } \\
\text { em reter cones mais pesados, enquanto } \\
\text { que mulheres com introito largo podem } \\
\text { achar dificuldade em reter, até mesmo, } \\
\text { cones mais leves } \\
\text { - Não devem ser utilizados em presença de } \\
\text { infecção vaginal, durante gestação ou } \\
\text { período menstrual }\end{array}$ \\
\hline $\begin{array}{l}\text { Eletroestimulação } \\
\text { de superfície } \\
\text { (EMG) }\end{array}$ & $\begin{array}{l}\text { - Os potenciais benefícios dos } \\
\text { eletrodos evidenciam uma } \\
\text { reduzida capacidade de invasão e } \\
\text { risco; e a facilidade com que os } \\
\text { pacientes podem utilizar essa } \\
\text { tecnologia para a prática } \\
\text { domiciliar }\end{array}$ & $\begin{array}{l}\text { - As contraindicações são: infecções } \\
\text { vaginais, diminuição da percepção } \\
\text { sensorial da vagina, infecção urinária, } \\
\text { arritmia cardíaca } \\
\text { - Adicionalmente, acesso ao método, } \\
\text { custo, durabilidade e a experiência do } \\
\text { profissional que avalia contração do MAP } \\
\text { na IUE }\end{array}$ \\
\hline Biofeedback & $\begin{array}{l}\text { Permite a conscientização de um } \\
\text { músculo pouco utilizado como o } \\
\text { elevador do ânus e leva à } \\
\text { aprendizagem, pela autocorreção }\end{array}$ & $\begin{array}{l}\text { - Adicionalmente, acesso ao método, custo, } \\
\text { durabilidade e a experiência do } \\
\text { profissional que avalia contração do MAP } \\
\text { na IUE }\end{array}$ \\
\hline
\end{tabular}

Abreviatura: MAP: músculo do assoalho pélvico; IUE: incontinência urinária de esforço

Fonte: Nascimento, SM

funcional visual medida pelo Spearman's rho $(\mathrm{p}<0,01)$. Cohen's Kappa apresentou $\kappa=0,37$, com um erro padrão de medida de 0,16 . Não houve diferença na contração muscular classificada entre as intensidades de força fraca, moderada, boa e forte, por meio da avaliação funcional visual, quando comparada ao perineômetro $(\mathrm{p}=0,66)$. $\mathrm{O}$ perimeômetro utilizado para avaliar o MAP com testereteste permitiu a confiança da velocidade de contração e resistência e bons parâmetros com o intervalo de confiança (IC) e desvio padrão (DP) (IC = 0,79-0,92 $\pm 1,39 \mathrm{~N} / \mathrm{s})$ e $298 \%$ para a atividade contração do MAP. A avaliação funcional visual indica que seus parâmetros de velocidade de contração e resistência por meio do perimeômetro possuem bom teste-reteste para avaliação do $\mathrm{MAP}^{23}$.

O estudo de validação EMG superfície da medida da força do MAP mostrou uma elevada correlação da pressão interna dos dados. Usando pontuação padronizada de correlação da pressão do MAP e do músculo intravaginal, foi $r=0,75$. Umas medidas repetidas da análise multivariada de variância demonstraram que o biofeedback visual e auditivo de
EMG durante contrações do MAP intravaginal mostrou que a pressão aumenta, quando comparado com os ensaios sem biofeedback ${ }^{20}$. Por sua vez, deu-se outro enfoque ao estudo que avaliou o MAP de indivíduos saudáveis por meio da EMG superfície, em que se verificou um coeficiente de variação de $21,61 \%$, indicando que essa variabilidade deve ser considerada em experimentos com uma condição inadequada para a normalização de procedimentos, tais como: EMG e o biofeedback na sessão reabilitação, ou normalização dos procedimentos por contração isométrica máxima voluntária ${ }^{24}$.

Baseado nesta revisão, conclui-se que os métodos de avaliação funcional e perineômetro foram escolhidos por serem os métodos eficazes e de fácil aplicabilidade, sendo por isso os mais utilizados nas práticas clínicas para elaboração do tratamento fisioterapêutico da IUE.

Além desse método, os cones vaginais apontaram, como as principais vantagens, a sua eficácia, o fato de ser fácil e simples de utilizar, sendo de uso privado e pessoal, permitindo a autoavaliação dos progressos e 
um incentivo à continuidade da sua realização ${ }^{22}$. A avaliação do MAP é o principal aspecto para que se possa montar o protocolo de tratamento. Portanto, é de fundamental importância que as pacientes submetidas à cirurgia de Wertheim-Meigs sejam encaminhadas ao fisioterapeuta, de preferência com especialidade em oncologia, para realizarem uma avaliação do assoalho pélvico com o intuito de prevenir ou tratar a IUE, otimizando assim a qualidade de vida das pacientes ${ }^{22}$, já tão sofridas pelo diagnóstico e tratamento de câncer do colo do útero.

Com relação a eleger um método para avaliação de força mais efetivo, ressalta-se que há poucas evidências que demonstrem a sua utilização na prática clínica, principalmente no setor público, o que requer ainda uma disponibilidade orçamentária para esse Serviço de Fisioterapia, não sendo possível realizar um estudo mais detalhado sobre o assunto em questão, tendo em vista a pouca existência de dados ou evidências científicas registradas. Embora os métodos de avaliação funcional visual e perineômetro para testar a força do MAP tenham mostrado uma tendência para ser método de escolha.

\section{AGRADECIMENTOS}

A autora agradece ao Instituto Nacional de Câncer, pela oportunidade do aprendizado e pela valorização do profissional de saúde, bem como à Dra. Maria de Fátima Bussinger Ferreira, pelo estímulo à realização deste estudo e pela informação da prática profissional. Meu apreço também à Dra. Neli Muraki Ishikawa pelo conhecimento científico compartilhado e à Profa. Heloísa Rocha de Alkimim, pela revisão bibliográfica.

\section{Potencial Conflito de Interesses: Nada a Declarar}

\section{REFERÊNCIAS}

1. Wingo PA, Cardinez CJ, Landis SH. Long-term trends in cancer mortality in the United States, 1930-1998. Cancer. 2003, 97 Suppl. 12: 3133-3275.

2. Abrams, P, Cardozo, L, Fall, M, Griffiths, D, Rosier, P, Ulmsten, U. et al. Standardisation of terminology of lower urinary tract function: report from the Standardisation Subcommittee of the International Continence Society. Am J Obstet Gynecol. 2002; 187 (1): 116-126.

3. Brandt, FT; Albuquerque, CDC; Arraes, AF; Albuquerque, GF; Barbosa, CD; Araújo, CM., et al. Influência do volume vesical na avaliação ultra-sonográfica da junção uretrovesical e uretra proximal. Radiol Bras. 2005; 38 (1): 33-36.
4. Fonseca ESM, Camargo ALM, Castro RA, Sartori MGF, Fonseca MCM, Lima GR, et al. Validação do questionário de qualidade de vida (King's Health Questionnaire) em mulheres brasileiras com incontinência urinária. Rev Bras Ginecol Obstet 2005; 27(5): 235-42.

5. Bernardes NO, Péres FR, Souza ELBL, Souza OL. Métodos de tratamento utilizados na incontinência urinária de esforço genuína: um estudo comparativo entre cinesioterapia e eletroestimulação endovaginal. Rev Bras Ginecol Obstet 2000; 22 (1): 49-54.

6. Contreras Ortiz O, Coya Nuñez F, Ibañez G. Evaluación funcional del piso pelviano femenino (classificación funcional). Bol Soc Latinoam Uroginecol Cir Vaginal 1994; 1(1): 5-9.

7. Hahn I, Milsom I, Ohlsson BL, Ekelund P, Uhlemann C, Fall M. Comparative assessment of pelvic floor function using vaginal cones, vaginal digital palpation and vaginal pressure measurements. Gynecol Obstet Invest 1996; 41(4):269-74.

8. Amaro JL, Moreira ECH, Gameiro MO, Padovani CR. Pelvic floor muscle evaluation in incontinent patients. Int Urogynecol J Pelvic Floor Dysfunct 2005a; 16 (5):352-4.

9. Hundley AF, Wu JM, Visco AG. A comparison of perineometer to brink scores for assessment of pelvic floor muscle strength. Int Urogynecol J Pelvic Floor Dysfunct 2005; 192 (5):1583-91.

10. Moreira ECH. Valor da avaliação propedêutica objetiva e subjetiva no diagnóstico da incontinência urinária feminina: correlação com a força muscular do assoalho pélvico. Rev Bras Ginecol Obstet 2000; 22 (9):597.

11. Belo J, Francisco E, Leite H, Catarino A. Reeducação do pavimento pélvico com cones de plevnik em mulheres com incontinência urinária. Acta Med Port 2005; 18 (2): 117-22.

12. Nagib ABL, Guirro ECO, Palauro VA, Guirro RRJ. Avaliação da sinergia da musculatura abdomino- pélvica em nulíparas com eletromiografia e biofeedback perineal. Rev Bras Ginecol Obstet 2005; 27 (4): 210-5.

13. Thompson DL. The national coverage decision for reimbursement for biofeedback and pelvic floor electrical stimulation for treatment of urinary incontinence. J Wound Ostomy Continence Nurs 2002; 29 (1): 11-9.

14. Morkved S, Bo K, FjortofT. Effect of adding biofeedback to pelvic floor muscle training to treat urodynamic stress incontinence. Obstet Gynecol 2002; 100 (4): 730-9.

15. Voorham-Van Der Zalm PJ, Pelger RCM, Stiggelbout AM, Elzevier HW, Nijeholt GABLÀ. Effects of magnetic stimulation in the treatment of pelvic floor dysfunction. BJU Int 2006; 5:1035-8.

16. Bo K, Sherburn M. Evaluation of female pelvic-floor muscle function and strength. Phys Ther 2005; 85 (3): 269 -82.

17. Weidner AC, Barber MD, Visco AG, Bump RC, Sanders DB. Pelvic muscle electromyography of levator ani and external anal sphincter in nulliparous women and women 
with pelvic floor dysfunction. Am J Obstet Gynecol 2000; 183 (6):1390-9.

18. Barbosa AMP, Carvalho LR, Martins AMVC, Calderon IMP, Rudge MVC. Efeito da via de parto sobre a força muscular do assoalho pélvico. Rev Bras Ginecol Obstet 2005; 27 (11): 677-82.

19. WRIGLEY, T. The use of vaginal weighted cones in the tratmente of genuine stress incontinence. Phys Ther 1990; 76 (8): 430-2.

20. Workman DE, Cassisi JE, Dougherty MC. Validation of surface EMG as a measure of intravaginal and intra-abdominal activity: implications for biofeedback-assisted Kegel exercises. Psychophysiology 1993 Jan; 30 (1): 120-5.

21. Amaro JL, Agostinho A.D, Trindade JCS, Lautenschlager MFM, Gameiro MOO. Eletroestimulação endovaginal e cinesioterapia no tratamento da incontinência urinária de esforço. J Bras Ginecol 1997; 107 (6): 189-95.

22. Bo K. Vaginal weight cones. Theoretical framework, effect on pelvic floor muscle strength and female stress urinary incontinence. Acta Obstet Gynecol Scand 1995; 74 (2): 87-92.

23. Morin M, Dumoulin C, Gravel D, Bourbonnais D, et al. Reliability of speed of contraction and endurance dynamometric measurements of the pelvic floor musculature in stress incontinent parous women. Neurourol Urodyn 2007; 26 (3):397-403.

24. Araujo RC, Duarte M, Amadio AC. On the inter- and intra-subject variability of the electromyographic signal in isometric contractions. Electromyogr Clin Neurophysiol 2000; 40 (4):225-9.

\section{Abstract}

Introduction: women suffering from cervical cancer in stages Ia, Ib, and IIa, and primary cervical adenocarcinoma are subjected to radical hysterectomy of Wertheim-Meigs that may evolve with some adverse events, including effort urinary incontinence (UI) due to weakness of the muscles of the pelvic floor. The assessment of the pelvic floor muscle strength has a decisive role in the physiotherapy treatment of UI. Objective: to describe the physical methods that can be used to evaluate the muscular strength of the pelvic floor in women with UI after the WertheimMeigs' surgery. Method: this article is a literature review, which searched for studies published in the databases of Medline, Pubmed, Scielo, Lilacs, and Bireme in the period of 1996 to 2007, in English and Portuguese. Conclusion: based on this review, it appears that the method of functional assessment, is efficient and easy to apply and, therefore it is the most widely method used in physical therapy assessments in clinical practice.

Key words: Floor pelvic; Incontinence would urinate of effort; Hysterectomy and Rehabilitation 